\title{
Editorial (zbf Heft 3, 2015)
}

\author{
F. Eder $\cdot$ H. Altrichter $\cdot$ A. Paseka
}

Online publiziert: 16 . Oktober 2015

(C) Springer Fachmedien Wiesbaden 2015

Dieses Heft vereint Blicke auf die Bildungsforschung mit Blicken in den konkreten Alltag von Schule und Jugendarbeit:

Veronika Kuhberg-Lasson, Katja Singleton und Ute Sondergeld widmen sich der Frage nach den Charakteristischen Merkmalen bildungswissenschaftlicher Projekte aus Deutschland und ihrer Entwicklung. Basis für ihre Analyse bilden Daten aus Projektdatenbanken. Dabei zeigen die Autorinnen auf, wie sich die Forschungsaktivitäten im Untersuchungszeitraum 1995 bis 2009 entwickelt haben. Bildungswissenschaftliche Forschung hat nicht nur quantitativ deutlich zugenommen, es gab auch Veränderungen hinsichtlich Finanzierung, inhaltlicher Schwerpunktsetzungen und empirischer Methoden.

Ina-Sophie Ristau, Sonja Meixner, Michaela Sixt und Jutta von Maurice geben Einblick in die Struktur des Nationalen Bildungspanels (NEPS) in Deutschland. Der Beitrag beschreibt Erhebungsdesign, Sampling, Fallzahlen und Panelverlauf der Startkohorte dieses für die Zukunft der Bildungsforschung in Deutschland extrem wichtigen Instruments und illustriert die Herausforderungen im Hinblick auf Rekrutierung, Stichprobenstabilität oder Datenschutz, die mit der Organisation und Durchführung einer solchen Längsschnittstudie verbunden sind. Zugleich werden aber auch die vielfältigen Forschungsmöglichkeiten sichtbar, die sich aus der Existenz eines Bildungspanels ergeben.

F. Eder $(\bowtie)$

Paris Lodron Universität Salzburg,

Salzburg, Österreich

E-Mail: ferdinand.eder@sbg.ac.at

H. Altrichter

Johannes Kepler Universität Linz,

Linz, Österreich

A. Paseka

Universität Hamburg,

Hamburg, Deutschland 
Drei weitere Beiträge richten den Fokus auf Lehrpersonen, Schüler und Schülerinnen sowie auf Jugendliche im sozialpädagogischen Kontext:

Steffen Amling beschäftigt sich mit Lernorientierungen von Grundschullehrer/ innen. Der Autor rekonstruiert Lernprozesse von Lehrkräften, wobei die den individuellen Lernprozessen zugrunde liegenden Haltungen, definiert als Art und Weise, wie Wissen erworben wird, im Zentrum stehen. Mittels dokumentarischer Methode kann er aufzeigen, dass sich in den Lernprozessen kollektive Formen des Kompetenzerwerbs ebenso zeigen wie schulstandortspezifische Merkmale der Berufspraxis.

In dem Artikel Populär oder abgelehnt? untersucht Imke Dunkake an deutschen Hauptschüler/innen die Zusammenhänge zwischen deren soziometrischen Positionen und soziodemografischen und sozialisationsrelevanten Merkmalen. Dabei zeigten sich Ergebnisse, die zum Teil von aus Grundschulen und Gymnasien bekannten Resultaten abweichen. Beispielsweise wurde kein Zusammenhang von Popularität und Schulleistungen gefunden: Schüler/innen aus Elternhäusern mit mehr Kulturkapital und höheren Bildungsabschlüssen stammen häufig aus der Gruppe der „,kontroversen" Schüler/innen.

Im Beitrag von Manuela Brandstetter erweitert sich der Blick von der Schule auf den Sozialraum. Kritisch wird das Sozialpädagogische Gestalten in kleinräumigen Gemeinden in den Fokus der Analyse gerückt. In einer Multiple Case Study werden Gruppendiskussionen und Interviews mit Jugendlichen, Kommunalpolitiker/ innen, Bildungsexpert/innen sowie Helfer/innen aus Jugendarbeit und Jugendhilfe, die in sechs Gemeinden im Feld lokaler Jugendarbeit aufgenommen wurden, konversationsanalytisch ausgewertet. In den verwendeten Sprachbildern und Redeweisen werden das jeweilige Verständnis von Jugendarbeit sichtbar sowie die aus den Benachteiligungseinschätzungen abgeleiteten kommunalen Gestaltungsversuche.

Die Rezensionen des vorliegenden Heftes betreffen zentrale Themen der Schulentwicklung. Sabrina Kulin bespricht den umfangreichen Sammelband von Marcus Pietsch, Barbara Scholand \& Klaudia Schulte (Hrsg.): Schulinspektion in Hamburg. Der erste Zyklus 2007 - 2013: Grundlagen, Befunde, Perspektiven (Münster/New York: Waxmann, 2015, 458 Seiten, broschiert, € 39,90, ISBN 978-3-8309-3278-9) und bietet einen guten Einblick in die Vielfalt der dort versammelten Beiträge. Josef Thonhausers Rezension über Mun Ling Lo: Lernen durch Variation. Implementierung der Variationstheorie in Schule und Bildungsforschung (Aus dem Englischen übersetzt von Gabriele Isak und Peter Posch. Münster und New York: Waxmann, 2015, 219 Seiten) kommentiert die in diesem Werk angebotenen Strategien zur Verbesserung und Weiterentwicklung des Unterrichts.

Ein Bericht über den diesjährigen Kongress der „Österreichischen Gesellschaft für Forschung und Entwicklung im Bildungswesen“ (ÖFEB) sowie Hinweise auf Publikationen von ÖFEB-Mitgliedern bilden wiederum den abschließenden Rahmen des Heftes. 